\title{
Predictores de LA ADAPTACIÓN SOCIOCULTURAL DE ESTUdIANTES UNIVERSITARIOS EXTRANJEROS EN ARGENTINA*
}

\section{PREDICTORS OF SOCIO-CULTURAL ADAPTATION OF FOREIGN UNIVERSITY STUDENTS IN ARGENTINA}

\author{
Alejandro Castro Solano** y María Laura lupano Perugini*** \\ ${ }^{*}$ Trabajo financiado por el Proyecto de Investigación Plurianual 112-200801-00473 del Consejo Nacional de \\ Investigaciones Científicas y Técnicas (CONICET). \\ **Doctor en Psicología. Miembro de la Carrera del Investigador del Consejo Nacional de Investigaciones Científicas y \\ Técnicas (CONICET). Director del proyecto plurianual Las competencias culturales y la predicción del éxito académico \\ y laboral de líderes y estudiantes migrantes. \\ Paraguay 5337, $5^{\circ}$ Piso Dpto. A - (1425) Ciudad Autónoma de Buenos Aires, República Argentina. \\ E-Mail: a.castro@fibertel.com.ar

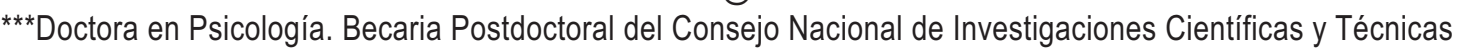 \\ (CONICET).
}

\begin{abstract}
RESUMEN
El interés para realizar esta investigación surgió a partir de que Argentina recibe un número creciente de estudiantes de otros países latinoamericanos, atraídos por el idioma, el prestigio académico y las condiciones económicas. Como resultado del contacto intercultural, los estudiantes atraviesan el proceso psicológico denominado aculturación, que implica cambios afectivos, comportamentales y cognitivos. En virtud de lo antedicho, este estudio se propuso identificar predictores de adaptación sociocultural en función de variables sociodemográficas, culturales y psicológicas de estudiantes que decidieron realizar sus estudios universitarios en Argentina. Participaron 216 estudiantes migrantes que se encontraban cursando diferentes carreras universitarias, el $57 \%$ eran mujeres $(n=124)$ y el $43 \%$ varones $(n=92)$, con un promedio de edad de 24.18 años $(D E=4.55)$. El 86\% $(n=186)$ había nacido en Latinoamérica. El restante 14\% provenía de países de Europa y Asia. El tiempo promedio de residencia en Argentina era de 3.31 años. Se emplearon diferentes instrumentos dise-
\end{abstract}

ñados ad hoc y/o adaptados para medir las variables involucradas en el estudio. A partir de los resultados obtenidos, no se pudo corroborar la influencia por parte de variables sociodemográficas (edad y tiempo de residencia). En cambio, entre las variables culturales se constató que percibir bajos niveles de discriminación y ansiedad intergrupal predice buenos niveles de adaptación sociocultural. En cuanto a las variables psicológicas, se corroboró que el apoyo social y la satisfacción percibida contribuyen a la adaptación sociocultural. En términos globales, las variables culturales y psicológicas predicen con mayor éxito la adaptación sociocultural en relación a las sociodemográficas.

Palabras clave: Predictores; Adaptación sociocultural; Estudiantes extranjeros; Discriminación; Ansiedad intergrupal; Apoyo social.

\section{ABSTRACT}

The interest of this research arises from that Argentina received a growing number of students 
from other Latin-American countries. International migration has risen exponentially in recent decades due to advances in technology and communications. According to the International Organization for Migration (OIM, 2010), the estimated number of international migrants in the world for that year was 214 million, doubling the previous two decades. Globalization has promoted exchanges among different cultures, but also caused the collision between different value systems, beliefs and customs that, in many cases, generates situations of confusion or discomfort in people (Furnham \& Bochner, 1986). Approximately 2.9 million people each year decide to make or complete their studies in other countries (OECD, 2008). Argentina receives an increasing number of students from other Latin-American countries, attracted by the language, the academic prestige of the local universities and the favorable economic conditions (Filmus, 2007). At the present, international students represent $1.6 \%$ of university students in Argentina. Therefore, Argentina is the fourth host country of America, after United States, Canada and Uruguay. As a result of intercultural contact, students go through the psychological process called acculturation, which involves affective, behavioral and cognitive changes. Taking into account the antecedents mentioned, this research proposed to identify predictors of socio-cultural adaptation based on socio-demographic, cultural and psychological variables, in international students who decided to make their university studies in Argentina. The participants of the study were 216 migrant students who were studying different careers. $57 \%$ were women $(n=124)$ and $43 \%$ males $(n=92)$ with an average age of 24.18 years $(S D=4.55) .86 \% \quad(n=186)$ were born in Latin-America. The remaining 14\% came from Europe and Asia. The average residence time in Argentina was 3.31 years. The reasons for their arrival in Argentina were diverse, $50 \%(n=108)$ of them considered that Argentina was a good place to study for its academic prestige and low costs. The remaining half was divided between family reasons $(n=47,21 \%)$, to have a different cultural experience $(n=30,14 \%)$, for student exchange $(n=19,9 \%)$ or others reasons $(n=13,6 \%)$. Regarding socio-economic status, the majority of respondents said they were in the middle class $(n=107,50 \%)$ and upper middle $(n=82,38 \%)$. Different instruments, designed ad hoc or adapted, were used to measure the variables involved in the study. To measure cultural variables were used instruments to assess perceived cultural distance, frequency of contact with foreigners and local habitants, intergroup anxiety and perceived discrimination. To measure psychological variables, were used instruments to assess social support and life satisfaction. Besides, were used instruments to assess the level of sociocultural adaptation. Based on results obtained, it could not be verified influence by socio-demographic variables (age and residence time). In contrast, among the cultural variables it was found that low levels of perceived discrimination an inter-grupal anxiety predicts good levels of sociocultural adaptation. Regarding psychological variables, it was confirmed that social support and perceived satisfaction contribute to this adaptation. In summary, cultural and psychological variables predict most successful socio-cultural adaptation than socio-demographic variables. One of the limitations of the study was the low inclusion in the sample of immigrants from countries with greater cultural distance. Futures studies can focuses on the analysis of the processes of adaptation with foreign students with language and customs more distant than Latin-American students. Other future research can study the perceptions of those who receive foreign students (host country) and analyze their relationship and influence on the perception of discrimination and prejudice from immigrants.

Key words: Predictors; Socio-cultural adaptation; International students; Discrimination; Intergrupal anxiety; Social support.

El contacto entre individuos de culturas diversas se ha convertido en un aspecto frecuente en la vida de las personas en el Siglo XXI (Bochner, 2006). Los individuos nacidos en una cultura, a menudo visitan otras naciones con fines laborales, humanitarios, de esparcimiento o de estudios (Ward, Bochner $\&$ Furnham, 2001). Si bien se trata de un fe- 
nómeno sumamente antiguo, las migraciones internacionales se han elevado exponencialmente en las últimas décadas debido a los avances tecnológicos y en las comunicaciones. Según la Organización Internacional para las Migraciones (OIM, 2010), el número estimado de migrantes internacionales en el mundo para ese año fue de 214 millones de personas, duplicando la cifra estimada de dos décadas previas. De este modo, la globalización ha favorecido el contacto entre miembros de diferentes culturas, pero al mismo tiempo ha provocado el choque entre distintos sistemas de valores, creencias y costumbres que, en muchos casos, genera situaciones de confusión o malestar en los individuos (Furnham \& Bochner, 1986).

En particular, el flujo reciente de estudiantes internacionales es el mayor registrado en la historia contemporánea. Aproximadamente, 2.9 millones de personas deciden cada año realizar o completar sus estudios en el extranjero (OECD, 2008). Tradicionalmente, los países que reciben un número mayor de estudiantes provenientes de otros lugares han sido Estados Unidos, Inglaterra, Alemania, Francia y Australia (Ward et al., 2001). Estos países, miembros de la OECD (Organización para la Cooperación y Desarrollo del Comercio), cuentan con aproximadamente 1.500.000 de alumnado extranjero en sus matrículas universitarias. Si bien las cifras son considerablemente menores en América Latina, este fenómeno se ha ido incrementando durante la última década. En particular, desde el año 2000, Argentina recibe un número cada vez mayor de estudiantes provenientes de otros países latinoamericanos, atraídos por el idioma, el prestigio académico de las universidades locales y las condiciones económicas favorables (Filmus, 2007). En la actualidad, los estudiantes internacionales representan un $1.6 \%$ de la matrícula universitaria argentina, convirtiendo a la Argentina en el cuarto país huésped de América, luego de Estados Unidos, Canadá y Uruguay.

Debido al carácter de residentes temporales en un país que no es el propio, los estudiantes internacionales han recibido la de- nominación de sojourners (Church, 1982). Esta categoría es también utilizada para describir a personas de negocios, técnicos expertos, cuerpos de paz y personal diplomático. Los sojourners migran a un contexto cultural diferente al suyo por un tiempo relativamente largo (de 6 meses a 5 años) con el fin de realizar una tarea determinada y con la supuesta intención de regresar a su país de origen una vez alcanzado el propósito de su viaje (Bochner, 2006; Ward et al., 2001). Es importante que los sojourners se adapten rápidamente a la nueva cultura para poder desarrollar efectivamente la tarea que desempeñan en el nuevo país (Church, 1982; Furnham \& Bochner, 1986).

Como resultado del contacto intercultural, los estudiantes extranjeros deben atravesar un proceso psicológico denominado aculturación, que implica cambios a nivel afectivo, comportamental y cognitivo (Sam $\&$ Berry, 2006). Es decir, un cambio en las actitudes hacia la propia identidad y hacia la cultura huésped. Cuando la aculturación se sostiene en el tiempo y los cambios permanecen estables se habla de un proceso de adaptación intercultural (Berry, 1997; Ward et al., 2001).

La adaptación puede ser psicológica o sociocultural (Ward, Fischer, Lam \& Hall, 2009). La psicológica está en relación con el bienestar experimentado como resultado del contacto cultural. La adaptación sociocultural involucra la puesta en marcha de habilidades sociales necesarias para funcionar adecuadamente en un entorno cultural complejo (Ward et al., 2001). Los predictores de la adaptación psicológica son el apoyo social percibido, las variables de la personalidad y los eventos vitales. En cambio, los factores que explican la adaptación sociocultural que involucran la resolución exitosa de problemas prácticos en la interacción con miembros de la cultura huésped son la distancia cultural, el conocimiento cultural, el grado de contacto con la cultura huésped y la discriminación percibida (Ward \& Kennedy, 1994). Si bien estas dos dimensiones están fuertemente relacionadas entre sí, muestran 
diferentes patrones de evolución y son explicados por distintos factores psicosociales (Ward et al., 2001).

Según Furnham y Bochner (1986), los estudiantes internacionales, además de enfrentar los problemas propios de quien migra de una cultura a otra (idioma, competencias culturales, discriminación y soledad) deben enfrentar dificultades similares a las de los estudiantes locales, como ser el ajustarse a la vida académica y los estresores propios de la transición de la escuela a la universidad. De este modo, pueden sentirse superados por las expectativas académicas propias de un contexto diferente al que fueron educados y experimentar fatiga cultural y otros síntomas físicos provocados por el constante esfuerzo cognitivo de operar en forma consciente y voluntaria para adaptarse (Berry, 2005; Mallinckrodt \& Leong, 1992). De este modo, como Ward y Kennedy (1994) sostienen, la experiencia de estudiar en el extranjero puede convertirse en uno de los sucesos más estresantes en la vida de las personas.

Desde la perspectiva de la Psicología Cultural, las investigaciones revelan que la estrategia aculturativa relacionada con la integración (es decir, mantener aspectos valorados de la cultura de origen e incorporar aspectos de la nueva cultura) se asocia a una mejor adaptación sociocultural y psicológica de los migrantes, según los estudios realizados en diferentes contextos culturales (Berry, Phinney, Sam \& Vedder, 2006; Zlobina, Basabe, Paez \& Furnham, 2008). Un reciente estudio realizado con estudiantes extranjeros en Argentina reveló que aquellas personas que empleaban un estilo integrativo como estrategia aculturativa, tenían un mejor rendimiento académico, se autopercibían más satisfechos y manifestaban una mejor adaptación tanto psicológica como sociocultural (Castro Solano, 2011a).

En relación a los factores individuales preexistentes al proceso de aculturación (sexo y edad) que actúan como moderadores del proceso de adaptación, las investigaciones realizadas arrojan resultados inconsistentes (Berry, 2006). En un estudio llevado a cabo con inmigrantes turcos en Canadá, se observó que las mujeres portaban niveles de adaptación sociocultural significativamente menores que los manifestados por los varones (Ataca \& Berry, 2002). Contrariamente, los resultados de investigaciones desarrolladas en otros contextos muestran que las mujeres obtienen niveles más altos de ajuste en relación a la adaptación práctica y de costumbres (Basabe, Zlobina \& Páez, 2004). La variable cultura de origen y el rol de la mujer en la esfera laboral, podrían estar cumpliendo un rol determinante en esos resultados.

Existe un consenso general acerca de que la adaptación intercultural es un proceso que ocurre secuencialmente (Bochner, 2006). Sin embargo, el debate se desarrolla en torno a si se trata de un proceso lineal o que presenta forma de U (Church, 1982; Ward et al., 2001). Estudios realizados con inmigrantes y con estudiantes extranjeros han demostrado que al llegar a la nueva sociedad, los individuos manifiestan altos grados de optimismo y entusiasmo. Sin embargo, estos sentimientos pronto son reemplazados por estrés y ansiedad debido a las dificultades propias de la vida cotidiana que las personas comienzan a enfrentar en un ambiente poco familiar. Gradualmente, a medida que las personas pueden afrontar esos problemas, adquieren nuevamente una sensación de seguridad y bienestar. Tanto la adaptación psicológica como la sociocultural tienden a ser bajas en un primer momento (Basabe et al., 2004; Ward, Okura, Kennedy \& Kojima, 1998; Zlobina et al., 2008). Con el paso del tiempo, disminuye el grado de sentimientos de ansiedad, soledad o tristeza como así también las dificultades socioculturales.

En relación a la adaptación sociocultural, la distancia cultural entre el país de origen y el de acogida, es uno de los principales factores que actúa como moderador entre el proceso de aculturación y los resultados del mismo (Searle \& Ward, 1990; Ward et al., 2001). La cultura de la que provienen los inmigrantes determina en gran medida la vivencia del choque cultural producido. La cercanía cultural conlleva un esfuerzo menor 
de adaptación debido a que favorece una comunicación fluida y reduce los niveles de ansiedad e incertidumbre (Church, 1982), en tanto aquellos individuos que provienen de sociedades más lejanas en términos culturales, deben esforzarse más por adquirir conocimientos y habilidades para desenvolverse con éxito en el país anfitrión (Ward \& Kennedy, 1994; Ward et al., 2001). En estudios realizados con inmigrantes se ha observado que la mayor fuente de dificultades para la adaptación fueron las diferencias en códigos, normas y valores culturales (Basabe et al., 2004; Zlobina et al., 2008). En la misma línea, los estudiantes extranjeros con mayor distancia cultural, muestran mayores dificultades para integrarse socialmente con sus pares locales (Redmond \& Bunyi, 1993), lo que a su vez implicaría una menor adaptación sociocultural. A mayor distancia cultural percibida, mayores son el estrés y las dificultades de adaptación tanto psicológica como sociocultural (Ward et al., 2001). Esta distancia muchas veces va acompañada de percepciones de discriminación. El grado de discriminación percibida es uno de los obstáculos más frecuentemente reportados por los inmigrantes para alcanzar una buena adaptación sociocultural (Ataca \& Berry, 2002; Basabe et al., 2004; Ward, 2004; Zlobina et al., 2008). Cuanto mayor es la discriminación que los inmigrantes perciben, mayores son las dificultades que ellos tienen para ajustarse a los problemas prácticos de la vida diaria en el país de acogida. Berry (2006) en un estudio realizado con estudiantes jóvenes, demostró que los estudiantes inmigrantes reportaron mayor índice de discriminación sobre la base de argumentos étnicos o culturales comparados con los estudiantes locales. Por lo tanto, la percepción de discriminación (haberse sentido discriminados por personas autóctonas) está vinculada a una menor orientación hacia el país de acogida, mayor hacia el de origen y una mayor actitud de aislamiento (Zlobina et al., 2008).

Estudios realizados desde la teoría del aprendizaje cultural han demostrado que los estudiantes internacionales tienden a perte- necer a tres redes sociales diferentes, cada una con una función psicológica particular (Bochner, McLeod \& Lin, 1977):

a.- El principal grupo está formado por compatriotas (personas provenientes del mismo país) y su función está asociada a brindar apoyo emocional y a reforzar la identidad y los valores de la cultura de origen.

b.- El segundo grupo está formado por miembros de la cultura huésped, cuya función es netamente instrumental y se basa en facilitar el cumplimiento de los objetivos académicos y profesionales.

c.- El tercer grupo está conformado por estudiantes internacionales, pero provenientes de países y culturas diferentes a la propia, cuya función es netamente recreacional, brindando un espacio de apoyo social mutuo por el hecho de compartir su carácter de extranjeros.

También es importante destacar el papel que juegan las redes sociales virtuales en el desarrollo del contacto del migrante con cualquiera de los grupos mencionados (Gómez Aguilar, 2004; Pantoja-Vallejo, Jiménez-Hernández \& Blanco López, 2012).

La satisfacción con los vínculos establecidos tanto con compatriotas como con estudiantes locales, se presenta como un fuerte predictor de adaptación psicológica (Searle \& Ward, 1990). Los estudiantes extranjeros más integrados con la comunidad local presentan mejor rendimiento académico, tienden a estar más satisfechos con su experiencia, se muestran menos ansiosos y reportan menores niveles de estrés y de dificultades sociales (Redmond \& Bunyi, 1993; Ward \& Kennedy, 1994). A pesar de los beneficios que trae el contacto con los locales, este tipo de red social es la menos desarrollada.

Desde la perspectiva de las teorías de estrés y afrontamiento, los investigadores también han considerado las interacciones sociales de los sojourners con miembros del país huésped (Ward et al., 2001) y focali- 
zándose en la cantidad y calidad de las interacciones, se ha observado que el apoyo social se presenta como un factor moderador de la satisfacción y bienestar psicológico de los estudiantes extranjeros (Ong \& Ward, 2005).

\section{EL PRESENTE ESTUdIO}

Un examen detallado de la literatura de investigación pone de manifiesto que existen predictores robustos de la adaptación sociocultural de los sojourners sin importar el tipo de grupo del que se trate, su país de origen y su lugar de destino (Ward, 1996; Ward \& Kennedy, 1994; Ward et al., 2001).

Entre las variables sociodemográficas se encuentra el tiempo de estancia en la cultura huésped, siendo la adaptación relativamente baja en ocasión del primer contacto intercultural y luego aumenta paulatinamente hasta alcanzar un plateau (Ward \& Kennedy, 1994; Ward et al., 1998). La edad es otra de las variables asociadas con la adaptación sociocultural, existiendo una relación inversa entre ambas. Si la persona que se acultura tiene menor edad, la misma se produce de modo más exitoso (Cheung, Chudek \& Heine, 2011; Yeh, 2003).

Entre las variables interculturales se encuentra el contacto interpersonal mantenido con miembros de la cultura huésped -tanto calidad como cantidad- (Ward \& Kennedy, 1994; Ward \& Rana-Deuba, 2000). Esto es, a mayor cantidad y calidad de contacto, mayor adaptación. La distancia cultural (grado de similitud entre la cultura de origen y la huésped) predice el grado de ajuste a la cultura receptora (Furnam \& Bochner, 1986; Ward \& Searle, 1991; Ward \& Kennedy, 1994). La idea subyacente es que a mayor distancia cultural, mayor es la necesidad de aprendizaje cultural para poder adaptarse. Aquellos que perciben mayor distancia cultural entre ambas culturas tienen mayores dificultades sociales para realizar la transición de un entorno cultural al otro (Searle \&
Ward, 1990; Ward \& Kennedy, 1994). El papel de la discriminación percibida fue menos estudiado. Los autores sostienen que la percepción de actitudes negativas de los sojourners por parte de los miembros de la cultura huésped ejerce una influencia negativa en la adaptación sociocultural (Navas et al., 2004; Ward, 1996; Ward \& Masgoret, 2005). En relación a la ansiedad intergrupal, los trabajos de Stephan, W.G. y Stephan, C. (1985) demostraron que una gama de sentimientos negativos tales como tensión, amenaza, desconcierto e incomodidad frente al contacto intercultural predecían mayores actitudes negativas hacia la cultura huésped y por lo tanto, una menor adaptación sociocultural (Stephan, W.G. \& Stephan, C., 1985; Stephan, Díaz-Loving \& Duran, 2000).

Si bien las variables de corte psicológico predicen en mayor medida la adaptación psicológica que la sociocultural, se consideró importante incluir en este estudio la satisfacción con la vida como predictor de este tipo de adaptación. Los estudios realizados por el grupo de Diener en la última década, demuestran que la satisfacción percibida (en términos amplios el bienestar psicológico) es un importante predictor de la salud física, de la longevidad, de las relaciones interpersonales y del funcionamiento psicológico óptimo de las personas (Diener $\&$ Biswas-Diener, 2008). En la misma línea se consideró importante incluir el apoyo social, ya que los estudios demuestran que es un factor moderador de la satisfacción y del bienestar psicológico de los estudiantes migrantes (Ong \& Ward, 2005).

Si bien la literatura es bastante consistente en cuanto a los predictores de adaptación sociocultural de los sojourners, muchas de las investigaciones citadas fueron realizadas con población mayormente neozelandesa y de otros países del sudeste asiático que emigraban hacia Nueva Zelanda.

Otro grupo de investigaciones citado hacía hincapié en los inmigrantes a los países centrales, tales como los Estados Unidos, Alemania, Francia, Inglaterra, Australia o Canadá (cf., Bochner, 2006; Ward et al., 2001). No se han encontrado estudios em- 
píricos de estudiantes migrantes desde y hacia contextos latinoamericanos.

Dado que como se comentó, la Argentina está recibiendo una afluencia cada vez mayor de estudiantes extranjeros, especialmente latinoamericanos hispanoparlantes (e.g., peruanos, ecuatorianos, venezolanos) se consideró importante replicar los estudios sobre predictores de adaptación sociocultural comentados en el contexto local.

\section{OBJETIVO E HIPÓTESIS}

El objetivo del trabajo realizado fue verificar si los predictores de adaptación sociocultural reseñados en la bibliografía internacional para diferentes grupos de soujourners y realizados en contextos culturales anglosajones, permiten predecir la adaptación sociocultural de estudiantes latinoamericanos migrantes que decidieron realizar sus estudios universitarios en la Argentina.

En función de los antecedentes reseñados, se hipotetiza un peso mayor de las variables estrictamente culturales, por sobre las psicológicas y las sociodemográficas, para la predicción de la adaptación sociocultural.

Por esta razón se utilizó como criterio una medida de la adaptación sociocultural breve (grado de ajuste al país huésped) y como predictores se incluyeron las variables sociodemográficas (edad y tiempo de residencia en el país huésped), culturales (distancia cultural percibida, contacto con extranjeros, connacionales y argentinos, ansiedad intergrupal y discriminación percibida) y psicológicas (apoyo social y satisfacción con la vida).

\section{MÉTodo}

\section{PARTICIPANTES}

La muestra empleada es de tipo no probabilística de sujetos voluntarios. Ellos eran alumnos universitarios provenientes de otro país. No se incluyeron extranjeros que viajaban solo para tomar cursos aislados, ni extranjeros que tuvieran más de 5 años de residencia en el país.

Participaron 216 estudiantes migrantes, que cursaban estudios en diferentes universidades públicas y privadas de la Ciudad de Buenos Aires.

El $57 \%$ eran mujeres $(n=124)$ y el $43 \%$ varones $(n=92)$. El promedio de edad fue de 24.18 años $(D E=4.55)$, sin variaciones significativas según sexo. En cuanto al estado civil, la mayor parte de ellos eran solteros $(n=204 ; 94 \%)$. En lo que respecta al país de procedencia, el $86 \%(n=186)$ había nacido en Latinoamérica y el 14\% restante en países de Europa y Asia.

Las razones de su arribo a la Argentina eran diversas, el 50\% $(n=108)$ consideraba que la Argentina era un buen lugar para estudiar por su prestigio académico y por sus bajos costos. El resto se repartía entre razones familiares $(n=47 ; 21 \%)$, para tener una experiencia cultural diferente $(n=30 ; 14 \%)$, por intercambio estudiantil $(n=19 ; 9 \%)$ o simplemente porque las circunstancias se dieron así o por azar $(n=13 ; 6 \%)$.

Con respecto al nivel socioeconómico, la mayor parte de los encuestados afirmó estar dentro de la clase media $(n=107,50 \%)$ y media alta $(n=82 ; 38 \%)$. El tiempo promedio de residencia en la Argentina era de 3.31 años.

\section{INSTRUMENTOS Y VARIABLES}

Se administraron varios instrumentos a los fines de estudiar las variables sociodemográficas, culturales y psicológicas.

\section{a.- Variables sociodemográficas}

Se solicitó a los participantes que contestaran una encuesta en la que debían consignar algunos datos sociodemográficos (sexo, edad, tiempo de residencia en el país huésped) y las razones de su arribo a la Argentina. 


\section{b.- Variables culturales}

1.- Distancia cultural percibida: Se evaluaron las diferencias percibidas entre la cultura de origen y la cultura argentina en función de algunas áreas relevantes para la adaptación sociocultural (amigos, comida, transporte, clima, etc.) mediante 18 ítemes diseñados sobre la base de la Escala de Adaptación Sociocultural de Ward y Kennedy (1999) y la Escala de Distancia Cultural de Zlobina y colaboradores (2008). Ambas pruebas fueron traducidas para el presente estudio.

Los participantes tenían que señalar si las conductas listadas eran similares o diferentes con respecto a su país de origen en una escala de 1 (extremadamente diferente) a 7 (extremadamente similar). Algunos de los ítemes propuestos incluían aspectos tales como amistades, comidas, normas y regulaciones, entre otros. Se calculó para cada participante un índice de distancia cultural percibida, que era la media de los ítemes listados. En virtud de la escala de puntuación del instrumento, un mayor puntaje indica menor distancia cultural percibida. La fiabilidad para esta escala fue igual a .90 .

2.- Frecuencia de contacto con extranjeros y locales: Sobre la base de las propuestas de Ward y colaboradores (2001) se diseñó una encuesta para evaluar la frecuencia de contacto de los estudiantes migrantes con otras personas de su país de origen, con otros extranjeros y con miembros de la cultura huésped.

Se preguntaba sobre la cantidad de amigos de su país, extranjeros (no de su país) $\mathrm{y}$ argentinos en una escala de 1 (ninguno)

1 Estas variabes fueron estudiadas con instrumentos que son versiones construidas ad hoc o adaptaciones realizadas en el marco del proyecto en el que se encuadra esta investigación. Los protocolos de los mismos pueden ser solicitados a los autores del presente estudio. a 5 (muchos). Si el encuestado pasaba el tiempo libre con personas de su país, extranjeros o argentinos en una escala de 1 (nunca) a 5 (siempre), tanto dentro como fuera del ámbito donde realizaba sus estudios.

Finalmente, se preguntaba acerca de la frecuencia de contacto total con miembros del país de origen, otros extranjeros y argentinos. Se calculó la media de los cuatro segmentos de preguntas y así se obtuvieron tres puntuaciones: (1) frecuencia de contacto con personas del mismo país $(\alpha=.86) ;(2)$ frecuencia de contacto con extranjeros $(\alpha=.85)$ y (3) frecuencia de contacto con argentinos $(\alpha=.88)$.

3.- Ansiedad intergrupal: Se utilizó una adaptación de la escala de Stephan, W.G. y Stephan, C. (1985) que evalúa ansiedad frente a grupos culturales diversos, modificada para los fines del presente estudio.

Básicamente se trata de adjetivos que indican si la persona tiene mayor o menor ansiedad ante el contacto con grupos culturales diversos. Se trabajó con 12 adjetivos y el evaluado debía responder en una escala con formato Likert de 7 puntos (de nada a mucho) el grado en que el contacto con extranjeros elicitaba las emociones positivas (e.g., confortable, confiado, seguro) y negativas (e.g., tenso, amenazado, ansioso) referidas por los adjetivos. A los fines de este estudio, en primer lugar se invirtieron los ítemes correspondientes a emociones positivas (ítemes $2,4,6,10,12)$ y luego se calculó una puntuación agregada (promedio de los ítemes) para todos los adjetivos. La fiabilidad obtenida fue igual a .85. Una mayor puntuación indica mayor ansiedad frente al contacto con extranjeros. Esta medida fue utilizada con éxito en estudios anteriores que evaluaban el ajuste percibido a la cultura huésped por diferentes tipos de soujournes (Castro Solano, 2011a, 2011b, 2011c; Castro Solano, Benatuil \& Laurito, 2012). 
4.- Discriminación percibida: Se adaptaron algunos ítemes del Proyecto Internacional de la Juventud Migrante (ICSEY) de Berry y colaboradores (2006) y así se confeccionó una encuesta de ocho ítemes que evaluaba la frecuencia de haber sido tratado injustamente, amenazado o no ser aceptado por pares, profesores o adultos por el solo hecho de pertenecer a un grupo cultural diferente.

Los participantes contestaban en una escala Likert con cinco opciones de respuesta (total acuerdo / total desacuerdo). Ejemplos de ítemes: "Pienso que en este país se trata injustamente a los extranjeros"; "Me insultaron o maltrataron por el sólo hecho de ser extranjero". La fiabilidad de esta prueba para la muestra de extranjeros calculada mediante el coeficiente Alpha fue igual a .88.

\section{c.- Variables psicológicas}

1.- Apoyo social: Se realizó una adaptación del Index of Sojourner Social Support (ISSS) de Ong y Ward (2005). El mismo consta de 18 ítemes que evalúan el apoyo social (instrumental y socioemocional) en estudiantes migrantes. La escala contiene ítemes que indagan sobre la percepción de poder contar con personas, por ejemplo, para hablar o ser escuchado, para hacer trámites, para estar acompañado, para recibir información necesaria para el contexto, etc. Se responde en una escala de 1 (nadie haría eso) a 5 (muchos harían eso). Se obtiene una sola puntuación (promedio de los ítemes) que indica el grado de apoyo social percibido. La fiabilidad para esta escala fue igual a .95 .

2.- Satisfacción con la vida: Se utilizó una versión adaptada al idioma español para uso con población argentina (Castro Solano, 2000) de la Satisfaction with Life Scale de Diener y colaboradores (1985). Es una escala de cinco ítemes con formato de respuesta Likert en 7 categorías y examina el grado de satisfacción global con la vida. La escala es utilizada internacionalmente para la evaluación del bienestar en tanto componente cognitivo de la satisfacción, por ejemplo: "En la mayoría de los sentidos, mi vida está cerca de mis ideales". Diferentes estudios empíricos han demostrado su validez y fiabilidad (Diener et al., 1985; Diener \& Larsen, 1991; Larsen, Diener \& Emmons, 1985). Se obtiene una puntuación promedio que indica el grado de satisfacción percibida por el evaluado.

Un estudio recientemente realizado sobre una muestra de argentinos adultos reveló una fiabilidad igual a .84 (Cosentino, 2011). La fiabilidad evaluada mediante el coeficiente Alpha de Cronbach para la muestra de los participantes de este estudio fue igual a .85.

3.- Adaptación sociocultural: La adaptación sociocultural es la habilidad social que tienen las personas para ajustarse al nuevo entorno sociocultural. Este medio resulta extraño en un primer momento y la adaptación futura deriva de la competencia para ejecutar interacciones exitosas con los aspectos culturales poco familiares o extraños. Se evaluó mediante cuatro ítemes en un formato Likert de cinco opciones de respuesta (e.g., grado de adaptación a la vida diaria, grado de ajuste al país huésped, calidad de la estancia, etc.). Se utilizó en el estudio una puntuación agregada (promedio de los cuatro ítemes). La fiabilidad evaluada mediante el coeficiente Alpha de Cronbach fue igual a .74. Esta medida fue utilizada con éxito en estudios anteriores en los que se evaluaba el ajuste percibido a la cultura huésped por diferentes tipos de soujournes (Castro Solano, 2011a, 2011b, 2011c; Castro Solano, Benatuil \& Laurito, 2012).

\section{Procedimiento}

Los instrumentos descriptos formaban parte de una batería de pruebas más amplia que se administró a estudiantes migrantes, estudiantes argentinos y población general de la ciudad de Buenos Aires con el propó- 
sito de obtener información sobre variables psicológicas relacionadas con el proceso de aculturación.

Los protocolos fueron administrados por estudiantes avanzados de la Carrera de Psicología de una universidad situada en la Ciudad Autónoma de Buenos Aires, los cuales se encontraban realizando sus prácticas profesionales en el área de investigación. Tenían como instrucción administrar la batería de pruebas a estudiantes extranjeros que habían iniciado sus estudios en la Argentina.

Los participantes no recibieron retribución alguna por su colaboración. Además, el cuadernillo que contenía las pruebas presentaba en su portada una introducción en la que se solicitaba el consentimiento del participante, se aseguraba el anonimato de los datos y su uso exclusivo para investigación.

En primer lugar, los administradores debían verificar que los sujetos encuestados tuvieran un manejo adecuado del idioma castellano por lo que se pidió que testearan la comprensión de las consignas iniciales y que los participantes completaran los protocolos en presencia de los administradores.

\section{ANÁLISIS DE LOS DATOS}

El análisis estadístico de los datos se realizó con el paquete SPSS versión 11.0.

En primera instancia se obtuvieron las medias, las desviaciones estándar y las intercorrelaciones de las variables introducidas en el estudio. Posteriormente se exploraron las relaciones entre las variables independientes y las dependientes mediante la realización de un análisis de regresión por pasos sucesivos. Se incluyeron como predictores las 10 variables señaladas previamente (dos sociodemográficas, dos psicológicas y seis variables culturales) y como criterio se consideró la adaptación sociocultural. No se incluyó la variable sociodemográfica sexo.

\section{Resultados}

Como se puede observar en los resultados expuestos en la Tabla 1, la mitad de las co- rrelaciones significativas presentan un tamaño del efecto de moderado $(r \geq .30)$ a amplio $(r \geq .50)$ (Cohen, 1992).

Las variables culturales que demostraron mayor correlación con la Adaptación sociocultural fueron la Distancia cultural percibida, el Contacto con argentinos, la Ansiedad intergrupal y la Discriminación percibida. Entre las psicológicas se encontraban la percepción de Apoyo social y la Satisfacción con la vida. Los estudiantes extranjeros que residían en países más similares al país huésped, que tenían contacto más frecuente con estudiantes locales, que demostraban menor ansiedad ante el contacto con personas de distinto bagaje cultural y que se sentían menos discriminados como extranjeros, fueron los que mostraron un mejor ajuste sociocultural. En la misma línea, los que registraban mayor Satisfacción con la vida y percibían un mayor Apoyo social, lograron una mejor Adaptación sociocultural. Dos de las correlaciones obtenidas presentaron tamaño del efecto amplio (Discriminación percibida y Adaptación sociocultural, Apoyo social y Adaptación sociocultural).

En un segundo momento se analizó la relación entre las variables predictoras y el criterio considerado (Adaptación sociocultural) mediante un análisis de regresión. Se utilizó el método de regresión hacia adelante (forward) para poder evidenciar aquellas variables que tenían más fuerza en la predicción del criterio (ver Tabla 2). De los 10 predictores ingresados en el modelo, cuatro de ellos explicaron el $49 \%$ de la variancia del criterio -Adaptación sociocultural $[F(4,212)=51.78, p<.001]$. En conjunto, las variables psicológicas (Apoyo social y Satisfacción percibida) y culturales (Ansiedad intergrupal y Discriminación percibida) explicaron casi la mitad de la variancia del criterio considerado $\left[R=.71 ; R^{2}\right.$ (ajustado $\left.)=.49\right]$.

Examinando los coeficientes de regresión estandarizados se observó que la Discriminación percibida y la Ansiedad intergrupal predicen de forma negativa la Adaptación sociocultural. La Satisfacción con la vida y el Apoyo social predecían asimismo la Adaptación sociocultural efectiva. De es- 
tas cuatro variables, la Discriminación percibida y el Apoyo social fueron las que tuvieron más peso sobre la predicción de la Adaptación sociocultural. Los estudiantes que percibieron poder adaptarse más exitosamente al país huésped, fueron aquellos que sentían un trato justo de sus pares y los que percibían conductas de ayuda, tanto instrumentales como emocionales. En un grado menor se trataba de estudiantes altamente satisfechos con la vida en general y que registraban mayor nivel de emociones positivas que negativas frente al contacto con grupos culturales diversos.

\section{Conclusiones}

El objetivo del estudio realizado era identificar los predictores de Adaptación sociocultural de estudiantes internacionales en Argentina. Los resultados obtenidos permitieron confirmar en parte la hipótesis planteada, ya que los hallazgos se encuentran en concordancia con los obtenidos en investigaciones previas a nivel internacional.

Se pudo corroborar que en la predicción de la Adaptación sociocultural las variables culturales y psicológicas juegan un papel central, en tanto que las sociodemográficas no juegan un rol preponderante. Según los resultados obtenidos, no se verificó que los estudiantes que presentaban mayor tiempo en el país lograran mejores niveles de adaptación. En parte esto puede explicarse por lo que mencionan algunos autores (e.g., Bochner, 2006; Church, 1982; Ward et al., 2001), quienes plantean que el proceso de adaptación ocurre secuencialmente y genera alternadas experiencias por lo que no parecería ser un proceso lineal. En la muestra estudiada sólo pudo corroborarse una relación significativa entre el tiempo de residencia y el contacto con argentinos, lo que implica que pasar mayor tiempo en el país de acogida favorece una mayor interrelación con los residentes locales.

En relación con lo antedicho, los resultados permiten afirmar que tener contacto con los residentes argentinos ayuda a lograr me- jores niveles de Adaptación sociocultural. Se pudo observar cómo los participantes del estudio dan más importancia a la red conformada por miembros de la cultura huésped, la cual para algunos autores (e.g., Bochner et al., 1977; Ong \& Ward, 2005) suele tener una función netamente instrumental. En este caso no parecería que los estudiantes tomasen esta red con esa finalidad sino, más bien, como fuente de apoyo social como sostienen Ward y colaboradores (2001), siendo el Apoyo social otra de las variables que se pudo verificar como predictora de la Adaptación sociocultural. Cabe aclarar que el $86 \%$ de los participantes provenían de países latinoamericanos. En relación con esto, Basabe y colaboradores (2004) argumentan que los países de Latinoamérica tienden a pertenecer a culturas más bien colectivistas que funcionan grupalmente, valoran las reuniones y el pasar tiempo juntos. En ese sentido, los argentinos nativos valoran el compartir actividades grupales, lo que puede favorecer la integración de estos inmigrantes. Los países de pertenencia de los estudiantes incluidos en este estudio presentan en su mayor parte, poca distancia cultural y por lo tanto, una mayor cercanía cultural que exige un menor esfuerzo de adaptación y favorece una comunicación más fluida (Church, 1982; Smith \& Bond, 1999; Ward et al., 2001). Evidentemente estos factores contribuyen a que los estudiantes que participaron en esta investigación sientan bajos niveles de discriminación y prejuicio hacia ellos y esto los ayude a desenvolverse y resolver problemas de su vida diaria. Estos resultados se encuentran en consonancia con los estudios previos destacados en la Introducción (e.g., Berry, 2006). Dentro de las variables culturales estudiadas, otra que logró predecir niveles de adaptación sociocultural es la Ansiedad intergrupal. Parecería que la cercanía cultural entre extranjeros y argentinos favorecería a que los primeros no experimenten tantas sensaciones de estrés y ansiedad al amoldarse a las costumbres del país de acogida.

Por último, las variables psicológicas incluidas en este estudio (Apoyo social y Satisfacción) parecen contribuir sustancial- 
mente para la adaptación sociocultural de los extranjeros. El hecho de que los extranjeros puedan sentirse satisfechos y contenidos, generando sentimientos de autoconfianza, favorece el logro de una correcta adaptación (Basabe et al., 2004).

A modo de síntesis, se puede destacar -de acuerdo a los resultados obtenidos- que las variables culturales y psicológicas predicen con mayor éxito la Adaptación sociocultural que las sociodemográficas. Lo antedicho corrobora parcialmente la hipótesis propuesta. Además, resulta relevante destacar que entre las variables culturales expuestas, las que mayor influencia presentan sobre el nivel de Adaptación sociocultural son la Discriminación percibida y la Ansiedad intergrupal, por lo tanto parece ser vital para que los estudiantes extranjeros logren adaptarse efectivamente y que no experimenten emociones negativas a la hora de contactarse con miembros de la cultura huésped, ni sientan que no serán aceptados. En relación a las variables psicológicas, ambas (Apoyo so- cial y Satisfacción) han demostrado ejercer influencia sobre el nivel de adaptación sociocultural. Estos resultados fueron confirmados por el modelo de regresión múltiple efectuado en este estudio.

En virtud de los comentarios expuestos, se puede destacar que una de las limitaciones del estudio que se informa es la baja inclusión en la muestra de inmigrantes pertenecientes a países de mayor distancia cultural. Si bien la muestra intenta representar los porcentajes diferenciales de estudiantes extranjeros que llegan a la Argentina, ya que en su mayor parte son latinoamericanos, puede ser de especial interés realizar un estudio que se focalice en el análisis de los procesos de adaptación de extranjeros con idiomas y costumbres distantes.

Otras futuras líneas de investigación pueden recaer en el estudio de las percepciones de quienes reciben a los extranjeros (país de acogida) y analizar su relación e influencia en la percepción de discriminación y prejuicio por parte de los inmigrantes. 
TABLA 1

MEDIAS, DESVIOOS ESTÁNDAR Y CORRELACIONES ENTRE LAS VARIABLES INTRODUCIDAS EN EL ESTUDIO

\begin{tabular}{|c|c|c|c|c|c|c|c|c|c|c|c|c|c|c|}
\hline Variables & $M$ & Rango & $D E$ & 1 & 2 & 3 & 4 & 5 & 6 & 7 & 8 & 9 & 10 & 11 \\
\hline 1.- Adaptación sociocultural & 3.51 & (1 a 5$)$ & .80 & - & & & & & & & & & & \\
\hline 2.- Edad & 24.18 & (17 a 48) & 3.80 & -.01 & - & & & & & & & & & \\
\hline 3.- Tiempo (en meses) & 28.14 & $(1-92)$ & 17.80 & .11 & .23 & - & & & & & & & & \\
\hline 4.- Distancia cultural percibida & 3.62 & (1 a 7$)$ & .87 & .24 & -.06 & .12 & - & & & & & & & \\
\hline 5.- Contacto extranjeros & 3.26 & (1 a 5$)$ & .97 & .03 & -.12 & -.09 & -.07 & - & & & & & & \\
\hline 6.- Contacto connacionales & 3.02 & (1 a 5$)$ & .86 & .08 & -.13 & -.11 & -.06 & .13 & - & & & & & \\
\hline 7.- Contacto argentinos & 3.61 & (1 a 5$)$ & .91 & .35 & -.06 & .23 & .26 & -.39 & .06 & - & & & & \\
\hline 8.- Ansiedad intergrupal & 2.97 & (1 a 7$)$ & .88 & -.37 & -.02 & .01 & -.22 & .15 & -.12 & -.24 & - & & & \\
\hline 9.- Discriminación percibida & 1.94 & (1 a 5$)$ & .71 & -.60 & -.02 & -.12 & -.28 & .13 & -.02 & -.35 & .32 & - & & \\
\hline 10.- Apoyo social & 3.46 & (1 a 5$)$ & .74 & .56 & -.13 & .02 & .17 & -.05 & .14 & .40 & -.33 & -.36 & - & \\
\hline 11.- Satisfacción & 5.10 & (1 a 7$)$ & 1.06 & .40 & .02 & .01 & -.04 & .15 & .07 & -.02 & -.16 & -.27 & .25 & - \\
\hline
\end{tabular}

En negrita: correlaciones estadísticamente significativas: $(r \geq|.16|, p<.01)$ 
TABLA 2

PREDICCIÓN DE LA ADAPTACIÓN SOCIOCULTURAL EN FUNCIÓN DE VARIABLES CULTURALES Y PSICOLÓGICAS

\begin{tabular}{l|cc|}
\hline \multicolumn{1}{|c|}{ Variables } & \multicolumn{2}{|c|}{ Adaptación sociocultural } \\
& $\beta$ & $t$ \\
\hline Discriminación percibida & -.38 & $6.79^{* * *}$ \\
Apoyo social & .31 & $5.52^{* \star *}$ \\
Satisfacción & .16 & $3.07^{* * *}$ \\
Ansiedad intergrupal & -.12 & $2.18^{*}$ \\
& & \\
& & \\
$R=.71 ; R^{2}$ (ajustado) $=.49$ & & \\
$* * * 0<.001$ & & \\
${ }^{*} p<.05$ &
\end{tabular}

\section{REFERENCIAS BIBLIOGRÁFICAS}

Ataca, B. \& Berry, J.W. (2002). Psychological, sociocultural, and marital adaptation of Turkish immigrant couples in Canada. International Journal of Psychology, 37(1), 13-26. http://dx.doi.org/10.1080/0020759014300013 5

Basabe, N., Zlobina, A. \& Páez, D. (2004). Integración socio-cultural y adaptación psicológica de los inmigrantes extranjeros en el País Vasco [Socio-cultural integration and psychological adaptation of immigrants in Pais Vasco]. País Vasco: Eusko Jaurlaritzaren Argitalpen Zerbitzu Nagusia.

Berry, J.W. (1997). Immigration, acculturation and adaptation. Applied Psychology: An International Review, 46(1), 5-68. http://dx.doi.org/1 0.1111/j.1464-0597.1997.tb01087.x
Berry, J.W. (2005). Acculturation: Living successfully in two cultures. International Journal of Intercultural Relations, 29(6), 697-712. http://dx.doi.org/10.1016/j.ijintrel.2005.07.013

Berry, J.W. (2006). Mutual attitudes among immigrants and ethnocultural groups in Canada. International Journal of Intercultural Relations, 30(6), 719-734. http://dx.doi.org/10.10 16/j.ijin trel.2006.06.004

Berry, J.W., Phinney, J., Sam, D. \& Vedder, P. (2006). Inmigrant youth: Acculturation, identity and adaptation. Applied Psychology: An International Review, 55(3), 303-332. http:// dx.doi.org/10.1111/j.1464-0597.2006.0025 6.x

Bochner, S. (2006). Sojourners. En D.L. Sam \& J.W. Berry (Eds.), The Cambridge handbook of acculturation psychology (pp. 181-197). New York, NY, US: Cambridge University Press.

Bochner, S., McLeod, B. \& Lin, A. (1977). Friendship patterns of overseas students: A 
functional model. International Journal of Psychology, 12(4), 277-294. http://dx.doi.org/10. 1080/00207597708247396

Castro Solano, A. (2000). Estilos de personalidad, objetivos de vida y satisfacción vital. Un estudio comparativo con adolescentes argentinos [Personality styles, life goals and life satisfaction. A comparative study with Argentinean adolescents]. Tesis Doctoral no publicada. España: Universidad Complutense de Madrid.

Castro Solano, A. (2011a). Estrategias de aculturación y adaptación psicológica y sociocultural de estudiantes extranjeros en la Argentina [Acculturation strategies, psychological and sociocultural adaptation of migrant students in Argentina]. Interdisciplinaria, 28(1), 1-16.

Castro Solano, A. (2011b). Virtudes humanas y adaptación psicológica y sociocultural de estudiantes extranjeros en Argentina [Human virtues, psychological and socio-cultural adaptation of migrant students in Argentina]. Acta Psiquiátrica y Psicológica de América Latina, 57(2), 99-107.

Castro Solano, A. (2011c). La evaluación de las competencias culturales de los líderes mediante el Inventario de Adaptación Cultural [Leader's cultural competences assessment with the Cultural Adaptation Inventory]. Anales de Psicología de la Universidad de Murcia, 27(2), 507517.

Castro Solano, A., Benatuil, D. \& Laurito, J. (2012). Evaluación de la inteligencia cultural de estudiantes internacionales en Argentina [Cultural intelligence assessment of international students in Argentina]. Acta Psiquiátrica y Psicológica de América Latina, 58(2), 95-103.

Cohen, J. (1992). A power primer. Psychological Bulletin, 112(1), 155-159. http://dx.doi.org/ 10.1037/0033-2909.112.1.155

Cosentino, A.C. (2011). Fortalezas del carácter en militares argentinos [Character strengths in
Argentine militaries]. Tesis Doctoral no publicada. Universidad de Palermo, Argentina.

Cheung, B., Chudek, M. \& Heine, S. (2011). Evidence for a sensitive period for acculturation: Younger immigrants report acculturating at a faster rate. Psychological Science, 22(2), 147-152. http://dx.doi.org/10.1177/09567976 10394661

Church, A. (1982). Sojourner adjustment. Psychological Bulletin, 91, 540-572. http://dx.doi.org/ $10.1037 / / 00$ 33-2909.91.3.540

Diener, E. \& Biswas-Diener, R. (2008). The science of optimal happiness. Boston: Blackwell Publishing.

Diener, E., Emmons, R., Larsen, R. \& Griffin, S. (1985). The Satisfaction with Life Scale. Journal of Personality Assessment, 49(1), 71-75. http://dx.doi.org/10.1207/s15327752jpa4901_ 13

Diener, E. \& Larsen, R. (1991). The experience of emotional well-being. En M. Lewis \& J.M. Haviland (Eds.), Handbook of emotions. New York: Guilford.

Filmus, D. (2007, julio). Sintesis del discurso del dia 10 de Julio de 2007 [Synthesis of speech on July 10, 2007]. Recuperado el 8 de Agosto de 2007 de http://www.me.gov.ar/spu/Notici as/Noticias_Universitarias_2007/noticias_ju lio_2007_estu_arg.htm

Furnham, A. \& Bochner, S. (1986). Culture shock: Psychological reactions to unfamiliar environments. London: Methuen.

Gómez Aguilar, A. (2004). Nuevas dinámicas interculturales en la esfera pública virtual de internet [New intercultural dynamics of internet virtual public sphere]. Sphera Pública, 4, 6985.

Larsen, R., Diener, E. \& Emmons, R. (1985). An evaluation of subjective well-being measures. Social Indicators Research, 17, 1-17. http://dx. doi.org/10.1007/BF00354108

Mallinckrodt, B. \& Leong, F.T. (1992). Social support in academic programs and family envi- 
ronments: Sex differences and role conflicts for graduate students. Journal of Counseling \& Development, 70(6), 716-723. http://dx.doi. org/ 10.1002/j.1556-6676.1992.tb02154.x

Navas, M., Pumares, O., Sánchez, J., García, M. C., Rojas, A.J., Cuadrado, I., Asensio, M. \& Fernández, J.S. (2004). Las actitudes de aculturación de autóctonos e inmigrantes en Almería a través del modelo ampliado de aculturación relativa. En D. Uclés \& J.F. Giménez (Eds.), Informe económico de la provincia de Almería, 2003. El reto de la inmigración [Economic report of the province of Almeria, 2003. The challenge of immigration] (pp. 120-140). Almería: Cámara de Comercio, Industria y Navegación de Almería.

OECD (2008). Migration. Recuperado el 30 de Noviembre de 2011 de http://www.oecd.org/s tatistics/

Ong, A. \& Ward, C. (2005). The construction and validation of a social support measure for sojourners: The Index of Sojourner Social Support (ISSS) Scale. The Journal of Cross-cultural Psychology, 36(6), 637-661. http://dx. doi.org/10.1177/0022022105280508

Organización Internacional de Migraciones. (2010). Informe sobre las migraciones en el mundo 2010. Futuro de la migración: Creación de capacidades para el cambio [Report on world migration 2010. The future of migration: Building capacities for change]. Publicación $N^{\circ} S P N$ 0013. Francia: Imprimerie Courand et Associes. Pantoja-Vallejo, A., Jiménez-Hernández, A. \& Blanco López, E. (2012). Diversidad cultural y redes sociales [Cultural diversity and social networks]. España: Joxman Editores.

Redmond, M.V. \& Bunyi, J. (1993). The relationship of intercultural communication competence with stress and the handling of stress as reported by international students. International Journal of Intercultural Relations, 17(2), 235-254. http://dx.doi.org/10.1016/0147-1767 (93)90027-6
Sam, D.L. \& Berry, J.W. (2006). The Cambridge handbook of acculturation psychology. New York, NY: Cambridge University Press.

Searle, W. \& Ward, C (1990). The prediction of psychological and sociocultural adjustment during cross-cultural transitions. International Journal of Intercultural Relations, 14(4), 449464. http://dx.doi.org/ 10.1016/0147-1767 (90) 90030-Z

Smith, P. \& Bond, M. (1999). Social psychology: Across cultures. Needham Heights, MA, US: Allyn \& Bacon.

Stephan, W.G. \& Stephan, C. (1985). Intergroup anxiety. Journal of Social Issues, 41, 157-176. http://dx.doi.org/10.1111/j.15404560.1985.tb01 134.x

Stephan, W.G., Diaz-Loving, R. \& Duran, A. (2000). Integrated threat theory and intercultural attitudes: Mexico and the United States. Journal of Cross-Cultural Psychology, 31, 240249. http://dx.doi.org/10.1177/00220221000 31002006

Ward, C. (1996). Acculturation. En D. Landis \& R. Bhagat (Eds.), Handbook of intercultural training (2da. ed., pp. 124-147). Newbury Park, CA: Sage.

Ward, C. (2004). Psychological theories of culture contact and their implications for intercultural training and interventions. En D. Landis, J. Bennet \& M. Bennett (Eds.), Handbook of intercultural training (pp. 185-216). Thousand Oaks, California: Sage.

Ward, C., Bochner, S. \& Furnham, A. (2001). The psychology of culture shock. London: Routledge.

Ward, C., Fischer, R., Lam, F.S.Z. \& Hall, L. (2009). The convergent, discriminant, and incremental validity of scores on a self-report measure of cultural intelligence. Educational and Psychological Measurement, 69(1), 85105.

Ward, C. \& Kennedy, A. (1994). Acculturation strategies, psychological adjustment and socio- 
cultural competence during cross-cultural transitions. International Journal of Intercultural Relations, 18, 329-343. http://dx.doi.org/10.1 016/0147-1767

Ward, C. \& Kennedy, A. (1999). The measurement of sociocultural adaptation. International Journal of Intercultural Relations, 23, 659-677.

Ward, C. \& Masgoret, A.M. (2005). Attitudes toward immigrants and immigration. Trabajo presentado en el New Directions, New Settlers, New Challenges, FRST End-users Seminar. Wellington.

Ward, C., Okura, Y., Kennedy, A. \& Kojima, T. (1998). Sociocultural adjustment during crosscultural transition. International Journal of Intercultural Relations, 22(3), 277-291. http://dx. doi.org/10.1016/S0147-1767(98)00008-X

Ward, C. \& Rana-Deuba, A. (2000). Home and host culture influences on sojourner adjustment. International Journal of Intercultural
Relations, 24, 291-306. http://dx.doi.org/10. 1016/S0147-1767(00)00002-X

Ward, C. \& Searle, W. (1991). The impact of value discrepancies and cultural identity on psychological and sociocultural adjustment of sojourners. International Journal of Intercultural Relations, 15, 209-225. http://dx.doi. org/10.10 16/0147-1767(91)90030-K

Yeh, C.J. (2003). Age, acculturation, cultural adjustment, and mental health symptoms of Chinese, Korean, and Japanese immigrant youths. Cultural Diversity and Ethnic Minority Psychology, 9(1), 34-48. http://dx.doi.org/10. 1037/1099-9809.9.1.34

Zlobina, A., Basabe, N., Paez, D. \& Furnham, A. (2008). Sociocultural adjustment of immigrants: Universal and group-specific predictors. International Journal of Intercultural Relations, 30(2), 195-211.

Consejo Nacional de Investigaciones Cientificas y Técnicas (CONICET) Universidad de Palermo Ciudad Autónoma de Buenos Aires República Argentina

Fecha de recepción: 8 de noviembre de 2012 Fecha de aceptación: 1 de agosto de 2013 
(ه) 